\title{
Quantum simulations of the superfluid-insulator transition for two-dimensional, disordered, hard-core bosons
}

\author{
Shiwei Zhang and N. Kawashima \\ Center for Nonlinear Studies and Theoretical Division \\ Los Alamos National Laboratory, Los Almaos, NM 87545 \\ J. Carlson and J.E. Gubernatis \\ Theoretical Division, Los Alamos National Laboratory, Los Almaos, NM 87545
}

(August 5, 2021)

\begin{abstract}
We introduce two novel quantum Monte Carlo methods and employ them to study the superfluid-insulator transition in a two-dimensional system of hardcore bosons. One of the methods is appropriate for zero temperature and is based upon Green's function Monte Carlo; the other is a finite-temperature world-line cluster algorithm. In each case we find that the dynamical exponent is consistent with the theoretical prediction of $z=2$ by Fisher and co-workers. 05.30.Jp, 02.70.Lq, 71.30.+h, 67.40.-w
\end{abstract}

Typeset using REVTEX 
Recently, the study of the superfluid-insulator phase transition in systems of disordered interacting bosons has attracted considerable attention. As discussed by Fisher and coworkers [1], this transition is associated with a zero-temperature transition from a superfluid to an insulating state as the strength of the disorder is increased. In addition to experimental studies in systems such as ${ }^{4} \mathrm{He}$ adsorbed in porous media, random magnets, Josephson junction arrays, exciton lifetimes in quantum well structures, vortices in bulk type-II superconductors, a huge amount of numerical work has been performed on models of these systems [2].

Computer simulations are a natural candidate for studying this transition. The results of the simulations to date, however, are somewhat surprising as they seem to indicate that slightly different models lead to different values of the relevant critical exponents. The most significant disagreement exists between the numerical work on the Vallain form of the disordered XY model [3, 4], which has produced a value of the dynamical exponent z consistent with the original prediction of $z=2$ given by Fisher and coworkers, and work on the hardcore boson model [5], which obtained $z=0.5$. This difference was suggested in the latter work to be the result of (unspecified) low-lying excitations that are not encompassed in the models studied by others. Recently, these claims have been questioned [2, 14,6]. Most of the numerical work on these dirty-boson systems has been based upon finite-temperature quantum Monte Carlo (MC) methods, although exact diagonalization [7] and large-N methods [8] have also been employed.

The aim of this Letter is to introduce novel zero- and finite-temperature quantum MC methods and to use them to study the superfluid-insulator transition in a hard-core dirtyboson system. As we discuss below, these two methods have important, distinct conceptual and practical advantages over the standard algorithms. Our zero-temperature algorithm is based on the Green's function Monte Carlo (GFMC) method [9] and allows one to compute ground-state properties directly and to perform scaling analysis in the spatial dimensions only. The finite-temperature algorithm employed is a new way to implement the world-line algorithm that can reduce the equilibration and auto-correlation times by several orders of 
magnitude.

Our Hamiltonian is the standard hard-core boson model with random disorder:

$$
H=-J \sum_{\langle i j\rangle}\left(b_{i}^{\dagger} b_{j}+b_{j}^{\dagger} b_{i}\right)+\sum_{i} V_{i} n_{i}^{2}
$$

where $J$ is the overlap integral between neighboring bosons, $\langle i j\rangle$ denotes a nearest neighbor pair of lattice sites, $b_{i}^{\dagger}$ and $b_{i}$ are the boson creation and destruction operators at lattice site $i$, and $n_{i}=b_{i}^{\dagger} b_{i}$ is the boson number operator which is restricted to have the eigenvalues of 0 or $1 . V_{i}$ is the on-site disorder and is assumed to be randomly and uniformly distributed over the interval $(-V, V)$. We consider $N_{b}$ bosons on a two-dimensional square lattice of $L \times L(=N)$ sites, with periodic boundary conditions in both spatial dimensions. In this work we restrict ourselves to half-filling $\left(\rho=N_{b} / N=1 / 2\right)$.

Two-dimensions occupies a special place in the theory of this model because of the predicted existence of a finite universal value of the dc conductivity [1]. Scaling theory also predicts that near the critical value of the disorder $V_{c}$ the singular part of the total (normal plus superfluid) compressibility $\kappa$ is expected to behave as $|\delta|^{\nu(z-d)}$, where $\delta=V-V_{c}$. If $z=d=2$, then near $V_{c}$ this quantity is finite and independent of system size. We observed this behavior in our zero-temperature simulations.

To apply the GFMC method [10], we define a kernel $K \equiv c-H$. In an occupation number basis $R$, all matrix elements $K\left(R, R^{\prime}\right)$ are readily obtained, and the constant $c$ is chosen such that these elements are non-negative. GFMC projects out the ground-state wavefunction $\psi_{0}$ by iterating the equation $\psi^{(t+1)}=K \psi^{(t)}$ from any positive initial function $\psi^{(0)}=\psi_{T}$. We can rewrite the equation in a form more appropriate for the MC process:

$$
\tilde{\psi}^{(t+1)}(R) \propto \sum_{R^{\prime}} \tilde{K}\left(R, R^{\prime}\right) \tilde{\psi}^{(t)}\left(R^{\prime}\right)
$$

where $\tilde{\psi}^{(t)}(R)=\psi_{T}(R) \psi^{(t)}(R)$ and $\tilde{K}\left(R, R^{\prime}\right)=\psi_{T}(R) K\left(R, R^{\prime}\right) \psi_{T}^{-1}\left(R^{\prime}\right)$. The trial wave function $\psi_{T}$ for the ground state serves as an importance function. In the MC process, $\tilde{\psi}^{(t)}$ at each $t$ is sampled by a finite ensemble of configurations (walkers) each carrying a weight. A walker at $R^{\prime}$ at time $t$ advances to time $t+1$ by sampling $R$ from $\tilde{K}\left(R, R^{\prime}\right) / \sum_{R} \tilde{K}\left(R, R^{\prime}\right)$, 
and determining a new weight from the product of the old weight and $\sum_{R} \tilde{K}\left(R, R^{\prime}\right)$. Splitting and combining procedures are applied at times to control the weights. A good $\psi_{T}$ can greatly reduce the fluctuations in the weight factors.

Once the iteration has reached the asymptotic limit, ground-state properties can be calculated from the distribution of configurations. The projection of the ground state here is free of the Trotter approximation or other systematic errors. The ground-state energy is given by $E=\left\langle H \psi_{T} / \psi_{T}\right\rangle$. The compressibility $\kappa=\left(N_{b} d^{2} E / d N_{b}^{2}\right)^{-1}$ can be computed directly from $E\left(N_{b}\right)$ by finite differences.

In GFMC, calculations of quantities other than the energy can usually be performed only approximately because the result of the simulation is a distribution of configurations proportional to $\psi_{T} \psi_{0}$. However, the superfluid density is a rather special property, being directly associated with the response of the system to moving boundaries: it is simply proportional to the long imaginary-time diffusion distance of the world-lines. By analogy with the arguments in [11], a diffusion displacement $\mathbf{D}$ can be defined:

$$
\mathbf{D}(\tau)=\frac{1}{L}\left[\sum_{i=1}^{N_{b}}\left(\mathbf{r}_{i}^{(t+\tau)}-\mathbf{r}_{i}^{(t)}\right)\right],
$$

where boundary crossings in the $x$ - and $y$-directions are included. It can then be shown that the superfluid density $\rho_{s}$ is given by

$$
\rho_{s}=\frac{c-E}{4 J} \lim _{\tau \rightarrow \infty} \frac{<\mathbf{D}^{2}(\tau)>}{\tau} .
$$

With GFMC, we are essentially examining the diffusion over a finite portion of the (infinite) zero-temperature paths. In the full path integral, the density of configurations would be proportional to the ground-state wave function $\psi_{0}$ at each end of this segment. We replace this condition at one end by an approximate one in which the density is proportional to $\psi_{T}$ instead of $\psi_{0}$. However, we emphasize that in the limit $(\tau \rightarrow \infty)$ that determines the superfluid density, these edge conditions have no effect, and the method is exact. We expect this method of computing the superfluid density to be quite valuable in many other applications, including the continuum. 
To extract the superfluid density, it is necessary to extrapolate diffusion distance to infinite $\tau$. Due to the local motion of the paths, even non-superfluid states will propagate a finite diffusion distance at large $\tau$. In order to project out this localized effect, we use the following to model the asymptotic diffusion

$$
\left\langle\mathbf{D}^{2}(\tau)\right\rangle / \tau=a^{\prime}+\left(b^{\prime} / \tau\right)\left[1-\exp \left(-c^{\prime} \tau\right)\right]
$$

where the constant $a^{\prime}$ determines the superfluid density and $b^{\prime}$ and $c^{\prime}$ are associated with "local" diffusion. The effects of higher $(\geq 2)$ orders in $1 / \tau$ are small and different functional forms with the same asymptotic expansion produce identical answers.

Our $\psi_{T}$ is the product of a one-body term and a Jastrow factor:

$$
\psi_{T}(R)=\Phi_{1} \exp \left[-\sum_{m, n=1}^{N_{b}} f\left(r_{m n}\right)\right],
$$

where $\Phi_{1}=\exp \left(-a \sum_{i} n_{i} V_{i}-b \sum_{<i j>} n_{i} V_{j}\right)$. The Jastrow factor contains long-range twobody correlations of the form

$$
f\left(r_{m n}\right)=\left\{\begin{array}{ll}
\alpha, & \text { if } r_{m n}=1 \\
\beta / r_{m n}^{\eta}, & \text { otherwise }
\end{array},\right.
$$

where $r_{m n}$ denotes the distance between bosons $m$ and $n$. The variational parameters, $a$, $b, \alpha, \beta$, and $\eta$, are optimized by minimizing the variance of the local energy. Due to the discrete nature of the problem, importance sampling with the long-range correlation in $\psi_{T}$ can be implemented in a very efficient way. We have verified that our results, in particular the superfluid density, remain unchanged except in statistical errors when these parameters deviate from their optimal values.

The results of our zero-temperature simulations are summarized in Figs. 1 and 2. We calculated the energy, compressibility, and superfluid density for system sizes of $4 \times 4$ up to $12 \times 12$. The disorder magnitude $V$ is the controlling parameter of the phase transition, and for each $V$, about 100 realizations of the disorder are studied. We verified that our results for the $4 \times 4$ system are in excellent agreement with the exact diagonalization studies [7]. Figure 1 presents the superfluid density as a function of disorder $V$ for various system sizes. The transition is evident by the changes in $\rho_{s}$ with system size for various $V$. 
To determine the precise scaling behavior, we used the following scaling relation for the superfluid density:

$$
\rho_{s}(\delta, L)=L^{-z} Y\left(L^{1 / \nu} \delta\right)
$$

where $Y$ is the scaling function. From this form, we see that when scaled with the correct value of $z$, curves for various $L$ will have a point of common intersection that marks the critical point $V_{c}(\delta=0)$. Results for $z=0.5,2.0$, and 3.0 are shown in Fig. 2. Clearly, $z=2$ is much favored over the other two values. A statistical analysis yields $V_{c}=9.9 \pm 0.4$, and $z=2.0 \pm 0.4$. As noted previously, if the $z=2$ scaling behavior is correct, the compressibility $\kappa$ should be finite and continuous in the region of the transition. We observed no statistically significant change in $\kappa$ in this region (see Fig. 1 inset), and found $\kappa \sim 0.10(1)$. We also determined the critical exponent $\nu$ to be $0.9 \pm 0.1$. This result is consistent with the lower bound of unity predicted by theory and with the value obtained for the quantum rotor model. It is not consistent with the estimate of $2.2 \pm 0.2$ found in [5].

To obtain evidence complementary to the zero-temperature calculation, we also performed finite-temperature MC simulations and employed a finite-size scaling analysis with two lengths, the lattice size $L$ and the inverse-temperature $\beta$.

To produce data, we extended a recently developed cluster algorithm [13 which is a form of world-line MC found to have smaller equilibration and autocorrelation times than the conventional implementation. The algorithm is based on two simple observations: in the absence of disorder, the hard-core boson model in two-dimensions maps onto a $S=1 / 2$ XY model [12] and the world-line algorithm for the $S=1 / 2 \mathrm{XY}$ model can be mapped onto the loop algorithm [14 for the 6-vertex model [15]. When used for world-lines, this method replaces the local movement of the world-lines by global changes of loops: the world-lines decompose into loops, each of which can be "flipped" with a probability of $1 / 2$. In the presence of disorder the flipping probability for a loop is

$$
1 /\left[1+\exp \left(\frac{1}{2} \Delta \tau \sum_{i \in \text { loop }}\left(1-2 n_{i}\right) V_{i}\right]\right.
$$


where $\Delta \tau$ is the imaginary-time spacing characteristic of the world-line method. This flipping probability and the loop construction [13,15] define our algorithm. Both the cluster and the conventional method suffer very long equilibration and autocorrelation times in strong disorder and at low temperature. These facts are the limiting factors of the finitetemperature simulations.

Because the winding number of each world-line is conserved in the conventional method, somewhat complicated procedures are necessary for measuring the superfluid density from the winding number variance [5]. In the new algorithm, however, winding number $\mathbf{W}$ is not conserved, so we can directly measure the superfluid density from $\rho_{s}=\left\langle\mathbf{W}^{2}\right\rangle / 4 J \beta$ where $\mathbf{W}$ is $\mathbf{D}(\tau)$ in (3) evaluated at $\tau=\beta$.

We performed simulations for two sets of space-time aspect ratios: one with $\beta / L^{2}=1 / 4$ and the other with $\beta / L^{1 / 2}=1 / 2$, corresponding to the two predictions $z=2$ and $z=1 / 2$. We used an open boundary condition in the temporal direction in the latter case to make it possible for the winding numbers to take non-integral values. Allowing this possibility is useful when the winding number fluctuations are much smaller than unity, as in the case of the second set of simulations. The number of MC steps used was up to $2 \times 10^{5}$ for the severest case. The number of random samples for the average is 32 to 128 . We carefully monitored the equilibration times by calculating a Hamming distance similar to the one adopted in [4] and found that as the system size or the inverse temperature increases, the equilibration time increases rapidly.

The finite-size scaling form for the finite-temperature superfluid density is

$$
\rho_{\mathrm{S}}(\delta, L, \beta)=\beta^{-1} \Upsilon\left(\delta L^{1 / \nu}, \beta / L^{z}\right)
$$

with the scaling function $\Upsilon$. The critical value $V_{c}$ should be the same as that obtained in the zero-temperature calculations. In Fig. 3, we present results corresponding to the two sets of simulations. Based on (10), curves should display a common crossing at $V=V_{c}$ if the chosen aspect ratio corresponds to the correct $z$.

We find that, contrary to the observation in [5], a common crossing point exists at $z=2$. 
In addition, the location of the estimated crossing point in (a) is $V_{c}=9.78 \pm 0.35$, in good agreement with the zero-temperature calculation. An apparent crossing occurs in (b) for $z=1 / 2$, but the crossing points have expanded to fill a larger region. Such "false" crossings can be difficult to diagnose given a limited range of system sizes and temperatures. Here, however, we note that the crossing region in (b) is very different from that found in the zero-temperature simulation, which should not be the case if $z=1 / 2$. Finally, we remark that an analysis of the results in (a) yields $\nu=0.90 \pm 0.13$.

In conclusion, this combination of zero- and finite-temperature algorithms have proven very powerful in studies of the superfluid-insulator transition in disordered hard-core bosons. The GFMC algorithm we have used produces exact results for the energy, compressibility, and superfluid density. As a zero-temperature method, it allows us to perform scaling in the spatial variables only, eliminating the need for any a priori assumptions. Our results for the compressibility and superfluid density convincingly demonstrate that $z=2$. This conclusion is strongly supported by the finite-temperature simulations, which are completely consistent with the zero-temperature calculations. The combination of zero- and finite-temperature simulations allow for a quite sensitive determination of the correct scaling behavior. Further details concerning our algorithms and our simulations will be reported elsewhere.

We acknowledge helpful conversations with D. Ceperley, M.P.A. Fisher, M.H. Kalos, R.T. Scaletter, N. Trevedi, and P. Weichman. We thank the Institute for Theoretical Physics (Santa Barbara) for its hospitality while part of the work was performed. Most of the simulations were performed on the computers at the National Energy Research Supercomputer Center and the Cornell Theory Center. The work of J.E.G. and S.Z. was supported in part by the High Performance Computing and Communication program of the Department of Energy. 


\section{REFERENCES}

[1] M.P.A. Fisher et al., Phys. Rev. B 40, 546 (1989).

[2] For a brief review of the numerical work and the physical systems, see G.T. Zimanyi, in Strongly Correlated Electronic Materials: the Los Alamos Symposium 1933, edited by K.S. Bedell, Z. Huang, D. Smeltzer, A. Balatsky, and E. Abrahams (Addision-Wesley, New York, 1994), to appear.

[3] E.S. Sørensen, et al., Phys. Rev. Lett. 69, 828 (1992).

[4] M. Wallin, et al., Phys. Rev. B 49, 12115 (1994).

[5] M. Makivic, et al., Phys. Rev. Lett. 71, 2307 (1993).

[6] P. Weichman, unpublished.

[7] K.J. Runge, Phys. Rev. B 45, 13136 (1992).

[8] Y.-H. Tu and P. Weichman, Phys. Rev. Lett. 73, 6 (1994).

[9] M.H. Kalos, Phys. Rev. 128, 1791 (1962); D.M. Ceperley and M.H. Kalos, in Monte

Carlo Methods in Statistical Physics, edited by K. Binder (Springer-Verlag, Heidelberg, 1979), chap. 4.

[10] J. Carlson, Phys. Rev. B 40, 846 (1989); N. Trivedi and D.M. Ceperley, Phys. Rev. B 41, 4552 (1990); S. Zhang and K.J. Runge, Phys. Rev. B 45, 1052 (1992).

[11] E.L. Pollack and D.M. Ceperley, Phys. Rev. B 36, 8343 (1987).

[12] T. Matsubara and H. Matsuda, Prog. Theor. Phys. 16, 416 (1956).

[13] N. Kawashima, et al., Phys. Rev. B, to appear; N. Kawashima and J.E. Gubernatis, Phys. Rev. Lett., to appear.

[14] H.G. Evertz, et al., Phys. Rev. Lett. 70, 875 (1993).

[15] U.-J. Wiese and H.-P. Ying, unpublished. 


\section{FIGURES}

FIG. 1. The superfluid density $\rho_{s}$ for different lattice sizes as a function of the strength of the disorder. The inset shows the corresponding compressibility $\kappa$ in the vicinity of $V_{c}$.

FIG. 2. Scaling plots assuming (a) $z=2$, (b) $z=1 / 2$, and (c) $z=3$. The common crossing

point is evident for $z=2$. For $z=1 / 2$, the crossing points systematically shift to the right as $L$ is increased. For $z=3$, the shift is to the left.

FIG. 3. Scaling plots with fixed aspect ratio assuming (a) $z=2$ and (b) $z=1 / 2$. 